\title{
Chryseobacterium piscium sp. nov., isolated from fish of the South Atlantic Ocean off South Africa
}

Correspondence

Hanli de Beer

hdebeer@cut.ac.za

\author{
Hanli de Beer, ${ }^{1}$ Celia J. Hugo, ${ }^{2}$ Piet J. Jooste, ${ }^{3}$ Marc Vancanneyt, ${ }^{4}$ \\ Tom Coenye ${ }^{5}$ and Peter Vandamme ${ }^{5}$
}

\author{
${ }^{1}$ School for Agriculture and Environmental Science, Central University of Technology: Free \\ State, 1 President Brandt Street, Bloemfontein 9300, South Africa \\ ${ }^{2}$ Department of Microbial, Biochemical and Food Biotechnology, University of the Free State, \\ Bloemfontein, South Africa \\ ${ }^{3}$ Department of Biotechnology and Food Technology, Tshwane University of Technology, \\ Pretoria, South Africa \\ ${ }^{4,5} \mathrm{BCCM} / \mathrm{LMG}$ Bacteria Collection ${ }^{4}$ and Laboratorium of Microbiology ${ }^{5}$, Ghent University, \\ K. L. Ledeganckstraat 35, Ghent B-9000, Belgium
}

Apart from being a source for several food-borne pathogens like Salmonella and Vibrio species and Listeria monocytogenes, raw fish, a protein-rich food with a neutral $\mathrm{pH}$ $(6 \cdot 6-6 \cdot 8)$ and high water activity, is a suspected source for microbial growth and deterioration. The combination of a high water content and the composition of the flesh enhance rapid spoilage in comparison with other types of raw meat (Sato, 2004). Psychrotolerant, Gram-negative bacteria dominate the spoilage flora, members of the family Pseudomonadaceae being major spoilage organisms (Forsythe, 2000). Rapid changes in the taxonomy of the family Flavobacteriaceae during the past decade and the description of Chryseobacterium joostei, isolated from milk (Hugo et al., 2003), initiated a long-term investigation into the incidence of yellow-pigmented colonies on raw fish. Chryseobacterium species inhabit water, soil and the clinical environment, but are also recovered from food sources, such as milk, meat, poultry and fish (Vandamme et al., 1994; Jooste \& Hugo,

The GenBank/EMBL/DDBJ accession number for the 16S rRNA gene sequence of Chryseobacterium piscium LMG $23089^{\top}$ is $\mathrm{AM040439.}$
1999; Hugo et al., 2003; Kämpfer et al., 2003; Li et al., 2003; Pavlov et al., 2004).

Potential Chryseobacterium isolates were obtained from fresh fish from the South Atlantic Ocean in the course of 1996 and were analysed by whole-cell protein electrophoresis. A cluster of four isolates collected in February (LMG 23086), June (LMG 23087) and July (LMG 23088 and LMG $23089^{\mathrm{T}}$ ) of that year differed from Chryseobacterium reference strains and prompted the taxonomic study reported herein. Reference strains from established Chryseobacterium species and related bacteria were available from the BCCM/ LMG Bacteria Collection (Ghent University, Ghent, Belgium). After growth for $24 \mathrm{~h}$ on tryptic soy agar (BBL), cells were harvested and whole-cell protein extracts were prepared and subjected to whole-cell protein electrophoresis as described by Pot et al. (1994). A densitometric analysis, normalization and interpolation of the protein profiles and a numerical analysis were performed by using the GelCompar software package (Applied Maths). The four isolates had virtually identical whole-cell protein profiles (the correlation level was above 0.96 ), which were most similar to those of the 

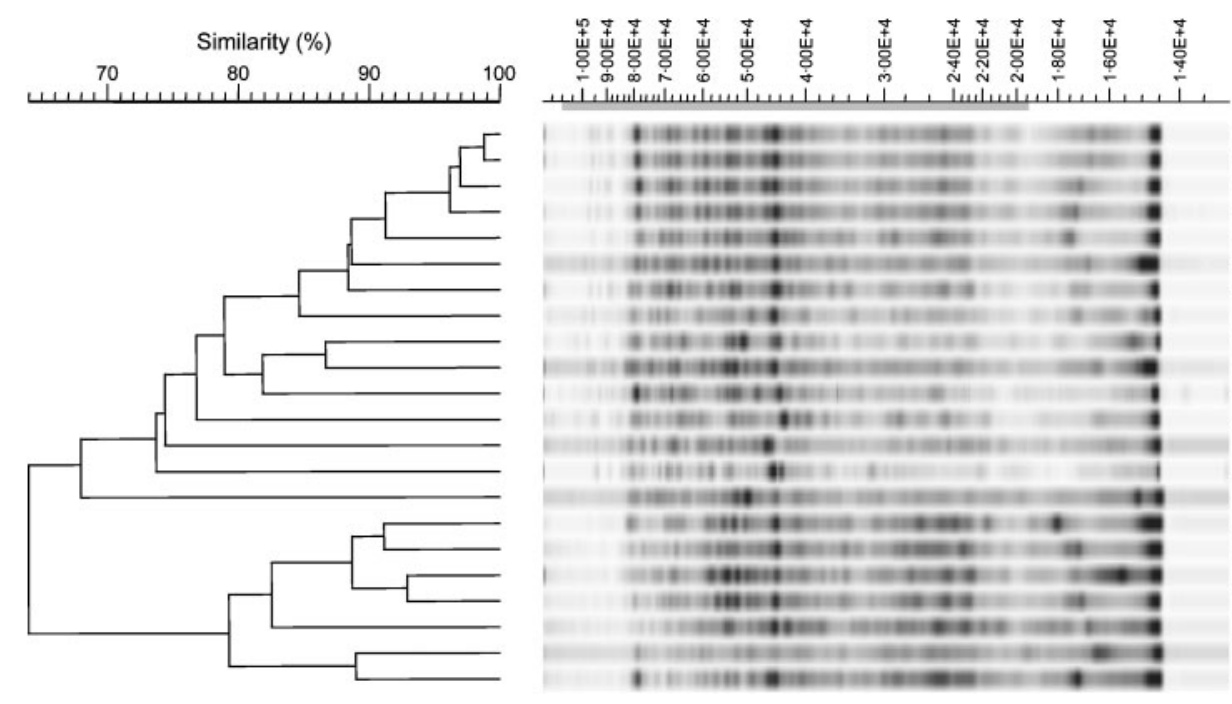

Chryseobacterium piscium
Chryseobacterium piscium
Chryseobacterium piscium
Chryseobacterium piscium
Chryseobacterium balustinum
Chryseobacterium formosense
Chryseobacterium indoltheticum
Empedobacter breve
Elizabethkingia meningoseptica
Chryseobacterium taichungense
Flavobacterium aquatile
Myroides odoratus
Weeksella virosa
Sphingobacterium spiritivorum
Elizabethkingia miricola
Chryseobacterium vrystaatense
Chryseobacterium gleum
Chryseobacterium indologenes
Chryseobacterium joostei
Bergeyella zoohelcum
Chryseobacterium defluvii
Chryseobacterium scophthalmum

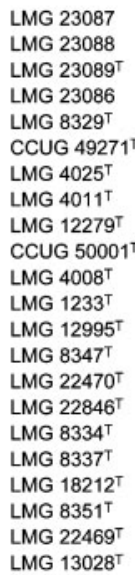

Fig. 1. Dendrogram derived from the unweighted pair group average linkage of correlation coefficients between the wholecell protein profiles of the novel strains (Chryseobacterium piscium sp. nov.) and reference strains of related taxa. Protein profiles were reproduced using the GelCompar software package. For convenience, the correlation coefficient is expressed as percentage similarity. The grey bar in the marker lane indicates the part of the protein profile that was used in the numerical analysis.

Chryseobacterium balustinum and Chryseobacterium indoltheticum type strains (correlation levels of 0.91 and 0.89 , respectively) (Fig. 1).

High-molecular-mass DNA of strain LMG $23089^{\mathrm{T}}$ and of Chryseobacterium reference strains was prepared according to Pitcher et al. (1989). In order to determine the phylogenetic position of this taxon, the $16 \mathrm{~S}$ rRNA gene sequence of LMG $23089^{\mathrm{T}}$ was determined as described by Willems et al. (2003) and analysed by using the BioNumerics 4.0 (Applied Maths) software package. A phylogenetic tree (Fig. 2) was constructed using the neighbour-joining method. The 16S rRNA gene sequence of LMG $23089^{\mathrm{T}}$ showed $99 \cdot 3,98.9$ and $97 \cdot 4 \%$ similarity to the corresponding gene sequences of the type strains of $C$. balustinum, Chryseobacterium scophthalmum and C. indoltheticum, respectively. Strain LMG $23089^{\mathrm{T}}$ and the C. balustinum and C. scophthalmum type strains formed a stable lineage

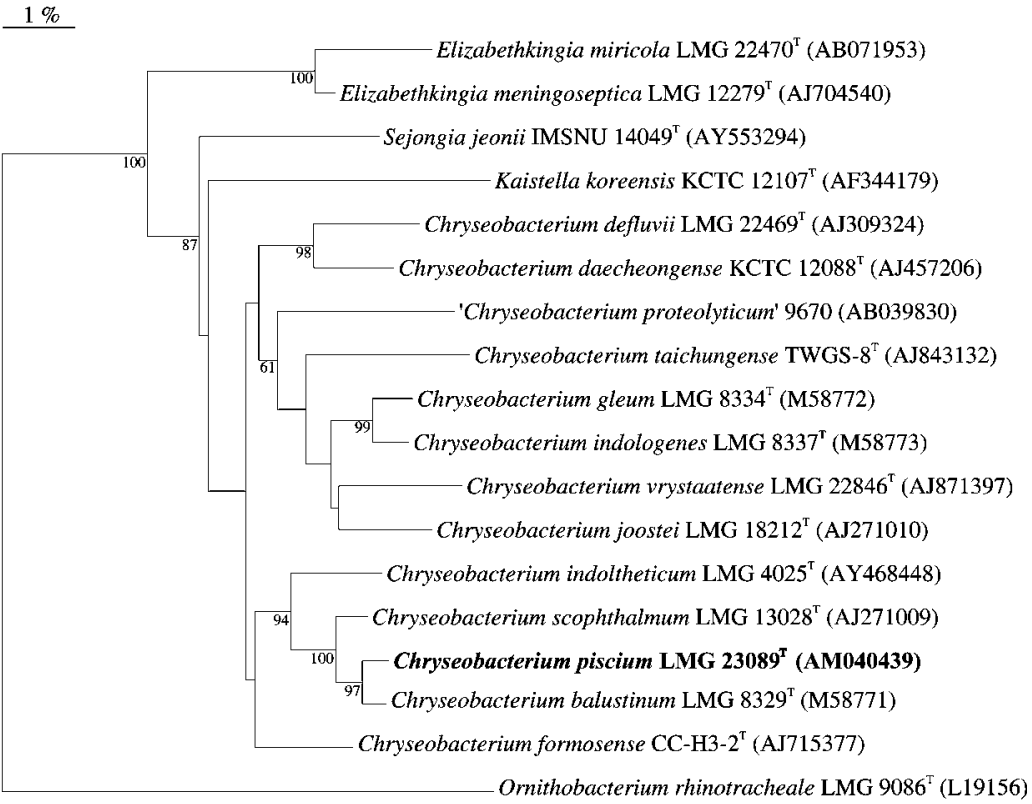

Fig. 2. Neighbour-joining phylogenetic tree of C. piscium sp. nov. LMG $23089^{\top}$ and related bacteria, based on 16S rRNA gene sequence comparisons. Bootstrap values above $60 \%$ (obtained with 1000 repetitions) are indicated. The $16 \mathrm{~S}$ rRNA gene sequence of Ornithobacterium rhinotracheale LMG $9086^{\top}$ was included as the outgroup. Bar, $1 \%$ sequence divergence. 
Table 1. Fatty acid compositions of Chryseobacterium and Elizabethkingia species

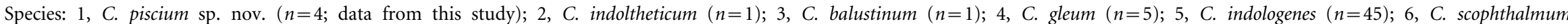

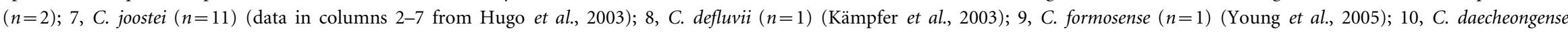

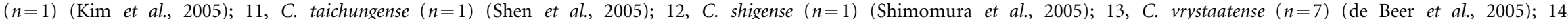

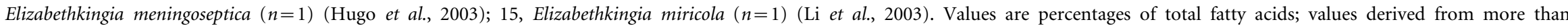
one strain are means \pm SD. Symbols: tr, trace amounts $(<1 \%)$; ND, not detected; NA, data not available; ECL, equivalent chain length (i.e. the identity of the fatty acid is unknown).

\begin{tabular}{|c|c|c|c|c|c|c|c|c|c|c|c|c|c|c|c|}
\hline Fatty acid & 1 & 2 & 3 & 4 & 5 & 6 & 7 & 8 & 9 & 10 & 11 & 12 & 13 & 14 & 15 \\
\hline iso- $\mathrm{C}_{13: 0}$ & $0 \cdot 9 \pm 0 \cdot 6$ & ND & $\operatorname{tr}$ & $\operatorname{tr}$ & $\operatorname{tr}$ & $\operatorname{tr}$ & $\operatorname{tr}$ & $2 \cdot 8$ & $3 \cdot 6$ & $1 \cdot 6$ & $\operatorname{tr}$ & NA & $1 \cdot 1 \pm 0 \cdot 4$ & $1 \cdot 4$ & $\operatorname{tr}$ \\
\hline ECL $13 \cdot 566$ & $\operatorname{tr}$ & $1 \cdot 7$ & $1 \cdot 6$ & $1 \cdot 2 \pm 0 \cdot 4$ & $2 \cdot 1 \pm 0 \cdot 7$ & $2 \cdot 9 \pm 0 \cdot 2$ & $1 \cdot 1 \pm 0 \cdot 2$ & $\operatorname{tr}$ & $\operatorname{tr}$ & $1 \cdot 5$ & $6 \cdot 7$ & NA & $1 \cdot 4 \pm 0 \cdot 3$ & $1 \cdot 5$ & $\operatorname{tr}$ \\
\hline anteiso- $\mathrm{C}_{15: 0}$ & $2 \cdot 7 \pm 1 \cdot 9$ & $5 \cdot 9$ & $\operatorname{tr}$ & $\operatorname{tr}$ & $\operatorname{tr}$ & $\operatorname{tr}$ & $\operatorname{tr}$ & $3 \cdot 2$ & $2 \cdot 1$ & $1 \cdot 0$ & $\operatorname{tr}$ & NA & $1 \cdot 7 \pm 0 \cdot 7$ & $2 \cdot 3$ & $\operatorname{tr}$ \\
\hline iso- $\mathrm{C}_{15: 0}$ & $38 \cdot 3 \pm 5 \cdot 0$ & $29 \cdot 4$ & $32 \cdot 3$ & $35 \cdot 4 \pm 2 \cdot 7$ & $34 \cdot 3 \pm 4 \cdot 9$ & $35 \cdot 0 \pm 0 \cdot 7$ & $34 \cdot 6 \pm 2 \cdot 0$ & $52 \cdot 2$ & $58 \cdot 5$ & $51 \cdot 2$ & $35 \cdot 4$ & $39 \cdot 7$ & $41 \cdot 8 \pm 1 \cdot 4$ & $41 \cdot 4$ & $33 \cdot 0$ \\
\hline iso- $\mathrm{C}_{15: 0} 3-\mathrm{OH}$ & $2 \cdot 4 \pm 0 \cdot 3$ & $2 \cdot 3$ & $2 \cdot 7$ & $2 \cdot 5 \pm 0 \cdot 1$ & $2 \cdot 6 \pm 0 \cdot 2$ & $2 \cdot 7 \pm 0 \cdot 1$ & $2 \cdot 9 \pm 0 \cdot 3$ & $2 \cdot 6$ & $1 \cdot 8$ & $2 \cdot 0$ & $4 \cdot 3$ & NA & $2 \cdot 7 \pm 0 \cdot 3$ & $3 \cdot 5$ & $3 \cdot 0$ \\
\hline $\mathrm{C}_{16: 0}$ & $1 \cdot 1 \pm 0 \cdot 2$ & $1 \cdot 0$ & $1 \cdot 6$ & $1 \cdot 4 \pm 0 \cdot 4$ & $\operatorname{tr}$ & $1 \cdot 2 \pm 0 \cdot 2$ & $\operatorname{tr}$ & $1 \cdot 3$ & $1 \cdot 5$ & $1 \cdot 8$ & $1 \cdot 3$ & NA & $1 \cdot 1 \pm 0 \cdot 3$ & $\operatorname{tr}$ & $2 \cdot 0$ \\
\hline $\mathrm{C}_{16: 0} 3-\mathrm{OH}$ & $1 \cdot 3 \pm 0 \cdot 3$ & $\operatorname{tr}$ & $1 \cdot 4$ & $1 \cdot 1 \pm 0 \cdot 1$ & $1 \cdot 0 \pm 0 \cdot 2$ & $1 \cdot 0 \pm 0 \cdot 1$ & $1 \cdot 2 \pm 0 \cdot 2$ & $\operatorname{tr}$ & $\operatorname{tr}$ & $\operatorname{tr}$ & $2 \cdot 6$ & NA & $1 \cdot 3 \pm 0 \cdot 3$ & $2 \cdot 2$ & $\operatorname{tr}$ \\
\hline iso- $\mathrm{C}_{16: 0} 3-\mathrm{OH}$ & $\operatorname{tr}$ & $1 \cdot 3$ & $\operatorname{tr}$ & $\mathrm{ND}$ & $\mathrm{ND}$ & $\mathrm{ND}$ & $\mathrm{ND}$ & $\operatorname{tr}$ & $1 \cdot 1$ & $\operatorname{tr}$ & $1 \cdot 4$ & NA & $\operatorname{tr}$ & $\operatorname{tr}$ & $8 \cdot 0$ \\
\hline ECL $16 \cdot 580$ & $1 \cdot 2 \pm 0 \cdot 2$ & $1 \cdot 3$ & $1 \cdot 3$ & $1 \cdot 7 \pm 0 \cdot 1$ & $1 \cdot 7 \pm 0 \cdot 2$ & $1 \cdot 5 \pm 0 \cdot 5$ & $1 \cdot 6 \pm 0 \cdot 1$ & ND & $1 \cdot 0$ & $1 \cdot 0$ & $1 \cdot 7$ & NA & $1 \cdot 2 \pm 0 \cdot 2$ & $1 \cdot 7$ & $\operatorname{tr}$ \\
\hline iso- $\mathrm{C}_{17: 0}$ & $1 \cdot 2 \pm 0 \cdot 2$ & $\operatorname{tr}$ & $1 \cdot 0$ & $1 \cdot 6 \pm 0 \cdot 6$ & $\operatorname{tr}$ & $\operatorname{tr}$ & $\operatorname{tr}$ & $2 \cdot 0$ & $2 \cdot 3$ & $3 \cdot 0$ & $\operatorname{tr}$ & NA & $\operatorname{tr}$ & $\operatorname{tr}$ & $1 \cdot 0$ \\
\hline iso- $\mathrm{C}_{17: 0} 3-\mathrm{OH}$ & $16 \cdot 2 \pm 3 \cdot 1$ & $14 \cdot 0$ & $16 \cdot 8$ & $21 \cdot 8 \pm 0 \cdot 3$ & $19 \cdot 2 \pm 1 \cdot 8$ & $16 \cdot 3 \pm 0 \cdot 1$ & $20 \cdot 1 \pm 1 \cdot 2$ & $14 \cdot 1$ & $10 \cdot 9$ & $15 \cdot 7$ & $22 \cdot 4$ & $19 \cdot 6$ & $15 \cdot 4 \pm 1 \cdot 8$ & $16 \cdot 3$ & $40 \cdot 0$ \\
\hline iso- $\mathrm{C}_{17: 1} \omega 9 c$ & $18 \cdot 7 \pm 2 \cdot 8$ & $25 \cdot 6$ & $27 \cdot 1$ & $20 \cdot 2 \pm 3 \cdot 9$ & $24 \cdot 2 \pm 3 \cdot 1$ & $24 \cdot 8 \pm 0 \cdot 4$ & $22 \cdot 9 \pm 1 \cdot 9$ & $4 \cdot 8$ & $4 \cdot 3$ & $7 \cdot 6$ & $8 \cdot 9$ & NA & $19 \cdot 7 \pm 2 \cdot 3$ & $7 \cdot 0$ & NA \\
\hline Summed feature $3^{*}$ & $10 \cdot 8 \pm 1 \cdot 3$ & $11 \cdot 2$ & $9 \cdot 2$ & $11 \cdot 8 \pm 0 \cdot 8$ & $11 \cdot 5 \pm 0 \cdot 3$ & $18 \cdot 1$ & $12 \cdot 1 \pm 1 \cdot 3$ & $8 \cdot 4$ & $6 \cdot 5$ & $10 \cdot 3$ & $13 \cdot 8$ & NA & $9 \cdot 1 \pm 2 \cdot 3$ & $18 \cdot 1$ & $12 \cdot 0$ \\
\hline
\end{tabular}

${ }^{*}$ Summed feature 3 comprises $\mathrm{C}_{16: 1} \omega 7 c, \mathrm{C}_{16: 1} \omega 7 t$ and/or iso- $\mathrm{C}_{15: 0} 2-\mathrm{OH}$. 
characterized by a bootstrap value of $100 \%$ (Fig. 2). The sequence similarity values with respect to the other type strains were below $97 \%$.

DNA-DNA hybridizations were performed with photobiotin-labelled probes in microplate wells as described by Ezaki et al. (1989), using an HTS7000 Bio Assay Reader (Perkin Elmer) for the fluorescence measurements. The hybridization temperature was $33^{\circ} \mathrm{C}$. In order to determine the $\mathrm{G}+\mathrm{C}$ content (mol\%) of strain LMG $23089^{\mathrm{T}}$, DNA was enzymically degraded into nucleosides as described by Mesbah et al. (1989). The obtained nucleoside mixture was then separated by HPLC using a Waters SymmetryShield C8 column regulated at $37^{\circ} \mathrm{C}$. The solvent was $0.02 \mathrm{M}$ $\mathrm{NH}_{4} \mathrm{H}_{2} \mathrm{PO}_{4}(\mathrm{pH} 4 \cdot 0)$ with $1.5 \%$ acetonitrile. Non-methylated $\lambda$ phage DNA (Sigma) was used as the calibration reference. The detector used was a Waters model 484 UVVIS absorbance detector set at $270 \mathrm{~nm}$. DNA-DNA hybridizations between strain LMG $23089^{\mathrm{T}}$ and the type strains of C. balustinum, C. scophthalmum and C. indoltheticum yielded binding values of 57,51 and $52 \%$, respectively. The DNA G + C content of LMG $23089^{\mathrm{T}}$ was $33.6 \mathrm{~mol} \%$, which corresponds with the range (34-38 mol\%) for the genus Chryseobacterium (Vandamme et al., 1994).

Whole-cell fatty acid components of the four strains were prepared from a loopful of well-grown cells that had been incubated for $24 \mathrm{~h}$ at $28^{\circ} \mathrm{C}$. Fatty acid profiles were generated under highly standardized conditions, as previously described by Vandamme et al. (1992). Separation and identification of esters were done with the Sherlock Microbial Identification System (MIDI, version 3.0). Mean percentages and standard deviations were calculated. Table 1 gives a summary of the fatty acid composition of the tested strains in comparison with those of the other Chryseobacterium reference strains. The mean percentages of the predominant fatty acids were as follows: iso- $\mathrm{C}_{15: 0}, 38 \cdot 3 \%$; iso- $\mathrm{C}_{17: 1} \omega 9 c$, $18 \cdot 7 \%$; iso- $\mathrm{C}_{17: 0} 3-\mathrm{OH}, 16 \cdot 2 \%$; and summed feature 3 , $10 \cdot 8 \%$.

A detailed phenotypic characterization of the four isolates was subsequently performed by using conventional biochemical techniques and the Biolog GN2 MicroPlate method. Data for other Chryseobacterium reference strains

Table 2. Phenotypic properties that differentiate C. piscium sp. nov. from other Chryseobacterium and Elizabethkingia species

Data were obtained in this study unless indicated otherwise. Species: 1 , C. piscium sp. nov. $(n=4) ; 2$, C. indoltheticum ( $n=1)$; 3 , C. balustinum $(n=1) ; 4$, C. gleum $(n=1) ; 5$, C. indologenes $(n=1) ; 6$, C. scophthalmum $(n=1) ; 7$, C. joostei $(n=1) ; 8, C$. defluvii $(n=1)$; 9, C. formosense $(n=1)$ (data from Young et al., 2005); 10, C. daecheongense ( $n=1)$ (Kim et al., 2005); 11, C. taichungense ( $n=1)$ (Shen et al., $2005) ; 12$, C. shigense $(n=1)$ (Shimomura et al., 2005); 13, C. vrystaatense $(n=36) ; 14$, E. meningoseptica $(n=1)$; 15, E. miricola $(n=1)$ (Li et al., 2003). Symbols: +, positive; w, weakly positive; -, negative; V, variable reaction; D, delayed; NA, no data available. Phenotypic tests were done as described by Hugo et al. (2003).

\begin{tabular}{|c|c|c|c|c|c|c|c|c|c|c|c|c|c|c|c|}
\hline Characteristic & 1 & 2 & 3 & 4 & 5 & 6 & 7 & 8 & 9 & 10 & 11 & 12 & 13 & 14 & 15 \\
\hline \multicolumn{16}{|l|}{ Growth on: } \\
\hline Cetrimide agar & - & - & - & - & + & - & + & - & NA & NA & $\mathrm{NA}$ & NA & + & + & + \\
\hline MacConkey agar & - & + & + & + & + & - & + & - & - & - & - & - & - & + & + \\
\hline \multicolumn{16}{|l|}{ Growth on nutrient agar at: } \\
\hline $5^{\circ} \mathrm{C}$ & + & + & + & - & - & + & + & - & - & - & - & + & + & - & NA \\
\hline $37^{\circ} \mathrm{C}$ & - & - & + & + & + & - & - & + & - & + & + & - & - & + & - \\
\hline $42{ }^{\circ} \mathrm{C}$ & - & - & - & - & + & - & - & + & - & - & - & - & - & + & NA \\
\hline $\begin{array}{l}\text { Growth on nutrient agar supplemented } \\
\text { with } 5 \% \mathrm{NaCl}\end{array}$ & + & - & - & - & - & - & - & - & NA & NA & NA & NA & - & - & NA \\
\hline \multicolumn{16}{|l|}{ Enzyme activity: } \\
\hline Urease & + & - & - & - & - & + & + & - & - & - & - & - & + & $\mathrm{D}$ & + \\
\hline Lecithinase & + & + & + & + & + & + & + & + & NA & NA & $\mathrm{NA}$ & NA & + & - & + \\
\hline Nitrate reduction & + & - & + & + & - & - & - & - & - & + & NA & - & - & - & - \\
\hline Nitrite reduction & - & - & - & + & - & - & - & - & - & - & NA & NA & + & + & - \\
\hline Starch hydrolysis & - & - & - & + & + & - & + & $\mathrm{W}$ & NA & + & NA & + & - & - & - \\
\hline Tween 80 hydrolysis & $-{ }^{*}$ & + & + & + & + & + & + & $\mathrm{NA}$ & - & - & NA & NA & + & - & + \\
\hline Tyrosine hydrolysis & - & $\mathrm{D}$ & - & + & - & + & + & + & NA & NA & NA & NA & - & $\mathrm{D}$ & - \\
\hline Phenylalanine deaminase & + & + & - & - & - & - & - & - & NA & NA & NA & NA & - & + & - \\
\hline \multicolumn{16}{|l|}{ Production of: } \\
\hline Hydrogen sulphide & - & + & - & - & - & - & - & - & - & - & - & - & $\mathrm{V}$ & - & + \\
\hline Indole & + & + & + & + & + & - & $\mathrm{W}$ & $\mathrm{W}$ & + & - & $\mathrm{w}$ & + & + & $\mathrm{D}$ & + \\
\hline
\end{tabular}

*Strains LMG 23087 and LMG 23088 tested positive. 
were obtained from a previous study (de Beer et al., 2005). A $24 \mathrm{~h}$ culture, incubated at $25^{\circ} \mathrm{C}$, was suspended in quarterstrength Ringer's solution to give a McFarland Barium Sulphate Standard 2 suspension. A battery of tests was selected to differentiate the species in the genus according to the methods described by Cowan (1974), MacFaddin (1980), Gerhardt et al. (1981) and Hugo et al. (2003), along with the Biolog GN2 MicroPlate, which was used according to the manufacturer's protocol. Test results are shown below in the species description and in Table 2, which includes the biochemical tests useful for the differentiation of the novel species from established Chryseobacterium species; specific phenotypic tests were performed as described by Hugo et al. (2003). The present data demonstrated that these four isolates from raw fish represent a novel species, for which we propose the name Chryseobacterium piscium.

\section{Description of Chryseobacterium piscium sp. nov.}

Chryseobacterium piscium (pis'ci.um. L. pl. gen. n. piscium of fish).

Colonies are shiny, yellow and translucent with entire edges. Cells are rod-shaped and the cell dimensions are approximately $1.0 \mu \mathrm{m}$ by $<0.5 \mu \mathrm{m}$ (Fig. 3). Cells are Gramnegative, catalase- and oxidase-positive and produce flexirubin pigment in the presence of $20 \% \mathrm{KOH}$. They produce an alkaline reaction in the oxidation-fermentation test. Colonies form after $24 \mathrm{~h}$ at $4,15,25$ and $32^{\circ} \mathrm{C}$. Very weak growth occurs on nutrient agar but not at 37 or $42^{\circ} \mathrm{C}$ or on MacConkey no. 3 (Oxoid CM115) agar. Grows in nutrient broth with a $5 \% \mathrm{NaCl}$ solution. Aesculin production occurs within $4 \mathrm{~h}$. Strains test positive for phenylalanine deaminase activity, gelatin hydrolysis, casein hydrolysis, lecithinase and phosphatase activity and produce urea from ammonia. Weak DNase activity occurs but there

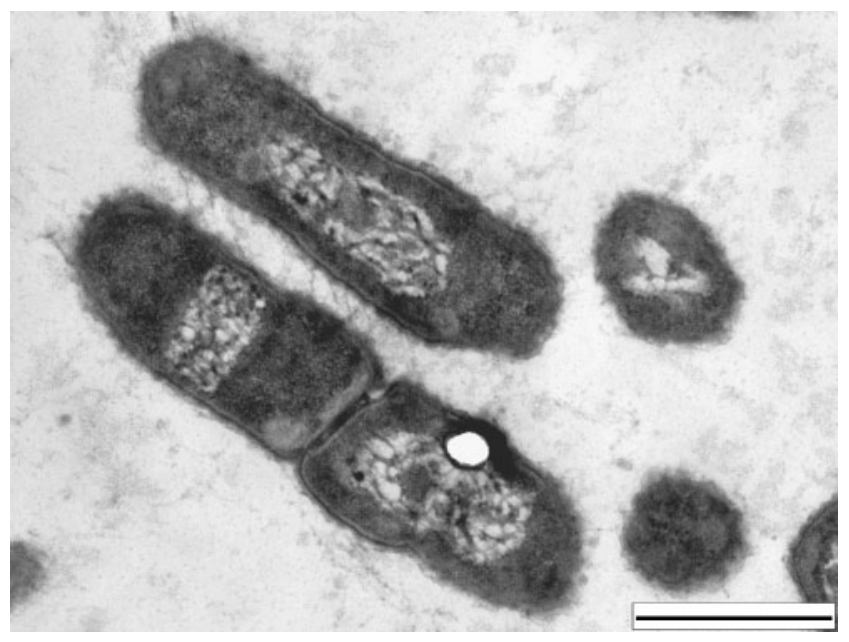

Fig. 3. Transmission electron micrograph of a cross-section of cells of C. piscium sp. nov. LMG $23089^{\top}$. Bar, $500 \mathrm{~nm}$. is no starch or tyrosine hydrolysis. Reaction on triple-sugar iron agar (Oxoid CM277) and $10 \%$ lactose is alkaline and strains do not produce $\mathrm{H}_{2} \mathrm{~S}$ from triple-sugar iron agar or SIM medium (Oxoid CM435). The ability of the strains to oxidize a panel of 95 different carbon sources was tested with the Biolog system: only four positive reactions were encountered in all four isolates, namely metabolism of gentiobiose, D-mannose, succinic acid monomethyl ester and acetic acid.

The type strain is $\mathrm{LMG} 23089^{\mathrm{T}}\left(=\mathrm{CCUG} 51923^{\mathrm{T}}\right)$. All currently known strains were isolated from fresh fish caught in the South Atlantic Ocean off the South African coastline during 1996. The production of urea and phenylalanine deaminase suggests that $C$. piscium may be involved in spoilage. Three additional isolates have been deposited in the BCCM/LMG Bacteria Collection as LMG 23086, LMG 23087 and LMG 23088.

\section{Acknowledgements}

We are indebted to the National Research Foundation, South Africa (H. de B.), and the Fund for Scientific Research - Flanders (Belgium) for a position as postdoctoral fellow (T. C.) and research grants (P.V.), respectively. We also wish to acknowledge Dr A. Jacoby (Central University of Technology, Bloemfontein, South Africa) and the Centre for Confocal and Electron Microscopy (University of the Free State, Bloemfontein, South Africa) for the transmission electron microscope image.

\section{References}

Cowan, S. T. (1974). Cowan and Steel's Manual for the Identification of Medical Bacteria, 2nd edn. Cambridge: Cambridge University Press.

de Beer, H., Hugo, C. J., Jooste, P. J., Willems, A., Vancanneyt, M., Coenye, T. \& Vandamme, P. A. R. (2005). Chryseobacterium vrystaatense sp. nov., isolated from raw chicken in a chickenprocessing plant. Int J Syst Evol Microbiol 55, 2149-2153.

Ezaki, T., Hashimoto, Y. \& Yabuuchi, E. (1989). Fluorometric deoxyribonucleic acid-deoxyribonucleic acid hybridization in microdilution wells as an alternative to membrane filter hybridization in which radioisotopes are used to determine genetic relatedness among bacterial strains. Int J Syst Bacteriol 39, 224-229.

Forsythe, S. J. (2000). The Microbiology of Safe Food. London: Blackwell Science.

Gerhardt, P., Murray, R. G. E., Costilow, R. N., Nester, E. W., Wood, W. A., Krieg, N. R. \& Phillips, G. B. (editors) (1981). Manual of Methods for General Bacteriology. Washington, DC: American Society for Microbiology.

Hugo, C. J., Segers, P., Hoste, B., Vancanneyt, M. \& Kersters, K. (2003). Chryseobacterium joostei sp. nov., isolated from the dairy environment. Int J Syst Evol Microbiol 53, 771-777.

Jooste, P. J. \& Hugo, C. J. (1999). The taxonomy, ecology and cultivation of bacterial genera belonging to the family Flavobacteriaceae. Int J Food Microbiol 53, 81-94.

Kämpfer, P., Dreyer, U., Neef, A., Dott, W. \& Busse, H.-J. (2003). Chryseobacterium defluvii sp. nov., isolated from wastewater. Int J Syst Evol Microbiol 53, 93-97.

Kim, K. K., Bae, H.-S., Schumann, P. \& Lee, S.-T. (2005). Chryseobacterium daecheongense sp. nov., isolated from freshwater lake sediment. Int J Syst Evol Microbiol 55, 133-138. 
Li, Y., Kawamura, Y., Fujiwara, N., Naka, T., Liu, H., Huang, X., Kobayashi, K. \& Ezaki, T. (2003). Chryseobacterium miricola sp. nov., a novel species isolated from condensation water of space station Mir. Syst Appl Microbiol 26, 523-528.

MacFaddin, J. F. (1980). Biochemical Tests for Identification of Medical Bacteria, 2nd edn. Baltimore: Williams \& Wilkins.

Mesbah, M., Premachandran, U. \& Whitman, W. B. (1989). Precise measurement of the $\mathrm{G}+\mathrm{C}$ content of deoxyribonucleic acid by high-performance liquid chromatography. Int J Syst Bacteriol 39, 159-167.

Pavlov, D., de Wet, C. M. E., Grabow, W. O. K. \& Ehlers, M. M. (2004). Potentially pathogenic features of heterotrophic plate count bacteria isolated from treated and untreated drinking water. Int J Food Microbiol 92, 275-287.

Pitcher, D. G., Saunders, N. A. \& Owen, R. J. (1989). Rapid extraction of bacterial genomic DNA with guanidium thiocyanate. Appl Microbiol 8, 151-156.

Pot, B., Vandamme, P. \& Kersters, K. (1994). Analysis of electrophoretic whole-organism protein fingerprints. In Chemical Methods in Prokaryotic Systematics, pp. 494-521. Edited by M. Goodfellow \& A. G. O'Donnell. London: Wiley.

Sato, M. (2004). Fish inspection. In Encyclopedia of Meat Sciences, vol. 1, pp. 474-482. Edited by W. K. Jensen, C. Devine \& M. Dikeman. Oxford: Elsevier.
Shen, F.-O., Kämpfer, P., Young, C.-C., Lai, W.-A. \& Arun, A. B. (2005). Chryseobacterium taichungense sp. nov., isolated from contaminated soil. Int J Syst Evol Microbiol 55, 1301-1304.

Shimomura, K., Kaji, S. \& Hiraishi, A. (2005). Chryseobacterium shigense sp. nov., a yellow-pigmented, aerobic bacterium isolated from a lactic acid beverage. Int J Syst Evol Microbiol 55, 1903-1906.

Vandamme, P., Vancanneyt, M., Pot, B. \& 10 other authors (1992). Polyphasic taxonomic study of emended genus Arcobacter with Arcobacter butzleri comb. nov. and Arcobacter skirrowii sp. nov., an aerotolerant bacterium isolated from veterinary specimens. Int J Syst Bacteriol 42, 344-356.

Vandamme, P., Bernardet, J.-F., Segers, P., Kersters, K. \& Holmes, B. (1994). New perspectives in the classification of flavobacteria: description of Chryseobacterium gen. nov., Bergeyella gen. nov., and Empedobacter nom. rev. Int J Syst Bacteriol 44, 827-831.

Willems, A., Fernández-López, M., Muñoz-Adelantado, E., Goris, J., De Vos, P., Martínez-Romero, E., Toro, N. \& Gillis, M. (2003). Description of new Ensifer strains from nodules and proposal to transfer Ensifer adhaerens Casida 1982 to Sinorhizobium as Sinorhizobium adhaerens comb. nov. Request for an Opinion. Int J Syst Evol Microbiol 53, 1207-1217.

Young, C.-C., Kämpfer, P., Shen, F.-T., Lai, W.-A. \& Arun, A. B. (2005). Chryseobacterium formosense sp. nov., isolated from the rhizosphere of Lactuca sativa L. (garden lettuce). Int J Syst Evol Microbiol 55, 423-426. 